\title{
Comparative Analysis of the Role of ERK1/2 Signaling Pathway in Regulating Cell Proliferation of Rat Liver Regeneration and Rat Acute Hepatic Failure
}

\author{
Cunshuan Xu (Corresponding author) \\ College of Life Science, Henan Normal University \\ Xinxiang 453007, Henan Province, China
}

$\&$

Key Laboratory for Cell Differentiation Regulation, HNP-STM

Xinxiang 453007, Henan Province, China

Tel: 86-373-332-6001Ｅ-mail: cellkeylab@126.com

Weiming Zhao

College of Life Science, Henan Normal University

Xinxiang 453007, Henan Province, China

Sa Wang

Key Laboratory for Cell Differentiation Regulation, HNP-STM

Xinxiang 453007, Henan Province, China

Wenbo Wang \& Yanjie Yang

College of Life Science, Henan Normal University

Xinxiang 453007, Henan Province, China

$\&$

Key Laboratory for Cell Differentiation Regulation, HNP-STM

Xinxiang 453007, Henan Province, China

Received: October 17, 2011

Accepted: November 4, $2011 \quad$ Published: December 31, 2011

doi:10.5539/jmbr.v1n1p55

URL: http://dx.doi.org/10.5539/jmbr.v1n1p55

This work is supported by the National Basic Research 973 Pre-research Program of China (NO. 2010CB534905)

\begin{abstract}
After injured or partially hepatectomy (PH), hepatocytes could rapidly enter cell cycle to compensate for the loss of liver tissue, which is regulated by lots of signaling pathway, including ERK1/2 signaling pathway. To compare the role of ERK1/2 signaling pathway in PH-induced liver regeneration (LR) with that in carbon tetrachloride-induced acute hepatic failure (AHF) at gene transcription level, Rat Genome 2302.0 array was used to detect gene expression profiles of above two processes, and bioinformatics and systems biology methods were applied to analyze the physiological activities uncovered by their profiles. The results showed that 165 genes were associated with fourteen paths of ERK1/2 signaling pathway, 161 genes were contained in the array above, and 46 genes were significantly expressed. Of them, 36 genes were the LR-specific genes, 24 genes were the AHF-specific, and 14 genes were the common genes. Synergy value of these genes was calculated using a
\end{abstract}


mathematical model established by our lab, and the results showed that the cell proliferation-promoting effect of path 4 was weaker than the control at $6 \mathrm{~h}$ of AHF occurrence, while those of paths 2,8 , and 9 at $12 \mathrm{~h}$ of LR and paths 2 and 6 at 72h of AHF were stronger. On the other hand, the cell proliferation-inhibiting effect of path 11 was stronger at $12 \mathrm{~h}$ of LR and AHF, but that of path 13 was weaker at 24h of LR. In conclusion, 46 genes related to seven paths of ERK1/2 signaling pathway regulates cell proliferation in both LR and AHF.

Keywords: Rat liver regeneration, Rat acute hepatic failure, ERK1/2 signaling pathway, Rat Genome 2302.0 Array, Cell proliferation

\section{Introduction}

The liver has a lot of important physiological functions and a strong ability to regenerate (Yokoyama et al., 2007). After injured or partially hepatectomy ( $\mathrm{PH})$, it can rapidly restore to the normal mass via proliferation and growth of the remnant liver, which is called liver regeneration (LR) (Higgins et al., 1931). This process is regulated by many signaling pathways including ERK $1 / 2$ signaling pathway. It was found that lots of physiological activities, including activation of cytokines and growth factors, synthesis and activation of transcription factors and enzymes, and synthesis and reconstruction of extracellular matrix were related to LR (Guo et al.,2006; Xu et al., 2010; Kant et al., 2006). In 2/3 hepatectomy-induced rat regenerating liver, hepatocytes were activated at $2 \mathrm{~h}$ after $\mathrm{PH}$, their transition from $\mathrm{G} 0$ phase to cell cycle occurred at 2-6h after $\mathrm{PH}$, their G1 phase, S phase, G2 phase, and M phase of first cell cycle were 6-12h, 12-24h, 24-30h, and 30-36h after $\mathrm{PH}$, respectively, and their second cell cycle was 36-66h after PH (Xu et al., 2010). Other types of hepatic cells also carried out proliferation and growth during this period. Then, the structure and function of the regenerating liver was re-established, and LR is completed within one week after PH.

Acute hepatic failure (AHF) is a kind of rapid liver cell necrosis or severe impairment of liver function which was induced by pathogenic factors. Lots of physiological activities including inflammatory and immune responses, signal transduction, metabolism, oxidation reduction, cell proliferation, differentiation and apoptosis occurred in AHF occurrence, which is regulated by many signaling pathways including ERK1/2 signaling pathway. Carbon tetrachloride $\left(\mathrm{CCl}_{4}\right)$-induced AHF was divided into injury phase (3-6h after $\mathrm{CCl}_{4}$ treatment), progressing phase (12-24h after $\mathrm{CCl}_{4}$ treatment) and recovery phase (48-72h after $\mathrm{CCl}_{4}$ treatment) (Mookerjee et al., 2007; Felipo et al., 2002; Cheng et al., 2010). At $3 \mathrm{~h}$ after $\mathrm{CCl}_{4}$ treatment, cytoplasm of hepatocytes became loose, and hydropic degeneration occurred to some. At $6 \mathrm{~h}$ after $\mathrm{CCl}_{4}$ treatment, hepatoytes underwent light vesicles-like steatosis, the hepatic sinusoids were hyperemic with obvious inflammatory cells infiltration. At $12 \mathrm{~h}$ after $\mathrm{CCl}_{4}$ treatment, hepatic lobes were damaged with obvious steatosis of hepatocytes, and hyperemia in hepatic sinusoids became more evident. At $24 \mathrm{~h}$ after $\mathrm{CCl}_{4}$ treatment, hepatic lobes injury increased with evident flake-shaped necrosis and monocytes and lymphocytes infiltration. In addition, hepatoytes underwent balloon sample degeneration, and hepatic sinusoids congestion became serious. At $72 \mathrm{~h}$ after $\mathrm{CCl}_{4}$ treatment, little lake-shaped but not bridge-shaped necrosis was observed in interlobular and portal area, hepatoyte proliferation and differentiation increased, and hepatic lobes recovered obviously.

ERK1/2 signaling pathway can regulate lots of physiological activities including cell proliferation and differentiation. After PH or drug injured, a large number of hepatocytes rapidly enter cell cycle to compensate for the loss of liver tissue, this process is regulated by lots of signaling pathway, including ERK $1 / 2$ signaling pathway. And ERK1/2 signaling pathway is composed of many proliferation-regulating paths. Our lab subdivided the ERK1/2 signaling pathway into 14 branch pathways which were named "paths", according to the path-path relevance and the role of the path, and according to the MEK1/2-activating factors, these paths were further grouped into four kinds. In the first kind of paths including path 1, 7-9 and 11, hormones and neurotransmitters activated G-protein, then MEK1/2. In the second kind of path, path 2, the concentration of Calcium ion varied, activating MEK1/2. In the third kind of paths, path 3-4, cytokines and growth factors combined with tyrosine kinase receptor, activating MEK1/2. In the forth kind of paths, path 5-6, extracellular matrix stimulated integrin-protein, activating MEK1/2. These factors assembled signals into MAPK kinase cascade, that is, MAPKKK (Raf) $\rightarrow$ MAPKK (MEK1/2) $\rightarrow$ MAPK (ERK1/2), activating ERK1/2. The activity of ERK1/2 was also regulated by other kinases (Path 10, 12-14) (Figure 2). Activated ERK1/2 enters the nuclear and phosphorylate lots of transcription factors and nuclear proteins to promote cell proliferation etc.

Several groups have reported that PH could activate ERK1/2 (Fausto et al., 2000; Huh et al., 2004; Leu et al., 2003), and ERK1/2 signaling could promote cell proliferation through regulating cell cycle progression in LR (Murata et al., 2007; Molecular And Cellular Biology et al., 1999; Rescan et al., 2001), and further promote the recovery of liver injury. Our lab has also compared expression profiles and role of rat LR-related genes with those of rat AHF-related genes at liver tissue level. However, it is unclear whether these paths participate in the 
regulation on liver regeneration, and how they do if any. To further explore the role of ERK $1 / 2$ signaling pathway in regulating rat LR and rat AHF at the gene transcription level, Rat Genome 2302.0 array was used to detect the expression changes of ERK1/2 signaling pathway-related genes in rat LR and in rat AHF, and bioinformatics and systems biology methods to analyze the influence of these changes on cell proliferation and apoptosis of rat LR and rat AHF occurrence.

\section{Materials and Methods}

\subsection{Rat 2/3 hepatectomy and rat acute hepatic failure}

Sprague-Dawley (SD) rats were obtained from the experimental Animal Center of Henan Normal University. They were bred in standard laboratory conditions (temperature $21 \pm 2{ }^{\circ} \mathrm{C}$, relative humidity $60 \% \pm 10 \%$, and $12: 12$ $\mathrm{h}$ light-dark cycle with lights on 8:00-20:00). 114 healthy SD rats, each weighting 230 $20 \mathrm{~g}$, were picked up and randomly divided into 9 partial hepatectomy groups, 9 operation control (OC) groups and one normal control (NC) group. Each group contained 6 ones. Rats in PH groups underwent $2 / 3 \mathrm{PH}$ as Higgins et al (1931) described, and they were sacrificed to isolate the liver tissues at 0,2, 6, 12, 24, 30, 36, 72, 120 and 168 hours after PH. In addition, 28 male rats, each weighting 250-320g, were picked up and randomly divided into 7 groups with 4 rats in each including 6 rat acute hepatic failure (AHF) group with 24 rats, and one control group with 4 rats. The rats were fed with standard rodent chow diet with free access to distilled water. The rats in AHF were fed with a single dose of $4 \mathrm{ml} / \mathrm{kg}$ carbon tetrachloride (CCl4) which was diluted 2:3 in sesame oil, and 4 livers were taken for use at each of the following time points: $3,6,12,24,48$ and 72 hours after CCl4 treatment. Livers of control group were taken at $0 \mathrm{~h}$. The whole handling procedure went in accordance with the current Animal Protection Law of China.

\subsection{Rat Genome 2302.0 microarray detection and data analysis}

Take $0.5 \mathrm{~g}$ liver tissues from the middle part of right lobe. Their total RNA was extracted from the frozen regenerating livers with Trizol reagent (Invitrogen Corporation, Carlsbad, California, USA) following its manufacturer's instructions and purified following the RNeasy mini protocol (Qiagen, Inc, Valencia, CA, USA), respectively. The quality of total RNA samples was assessed by measuring the optical density at $260 / 280 \mathrm{~nm}$ and agarose electrophoresis $(180 \mathrm{~V}, 0.5 \mathrm{~h})$ with a $2: 1$ ratio of $28 \mathrm{~S}$ rRNA to $18 \mathrm{~S}$ rRNA intensity. As a template, $5 \mu \mathrm{g}$ of total RNA was used to synthesize the first strand of cDNA using SuperScript II RT (Invitrogen Corporation, Carlsbad, CA), and T7-oligo dT(24) (W.M. Keck Foundation, New Haven, CT) as the primer. Second strand synthesis was performed with the Affymetrix cDNA single-stranded cDNA synthesis kit. The cDNA product was purified following the cDNA purify protocol. Twelve $\mu \mathrm{L}$ purified cDNA served as a template for the production of biotin-labeled cRNA transcript using the GeneChip in vitro Transcript Labeling Kit (ENZO Biochemical, New York, NY). The labeled cRNA was purified using the RNeasy mini columns (Qiagen, Valencia, CA) [7]. The concentration, purity and quality of cDNA and cRNA were assessed as above. Fifteen $\mu \mathrm{L}$ cRNA $(1 \mu \mathrm{g} / \mu \mathrm{L})$ was incubated with $6 \mu \mathrm{L} 5 \times$ fragmentation buffer and $9 \mu \mathrm{L}$ RNase free water for $35 \mathrm{~min}$ at $94{ }^{\circ} \mathrm{C}$, and digested into 35-200 bp cRNA fragments. The prehybridized rat genome 2302.0 microarray was put into a hybridization buffer prepared following the Affymetrix protocol, and hybridized in a rotating chamber $\left(60 \mathrm{rpm}, 16 \mathrm{~h}, 45^{\circ} \mathrm{C}\right)$. Arrays were washed to remove the superfluous hybridization buffer, stained in GeneChip fluidics station 450 (Affymetrix Inc., Santa Clara, CA, USA), scanned with a GeneChip scanner 3000 (Affymetrix Inc., Santa Clara, CA, USA) to obtain images (Xu et al., 2008).

\subsection{Quantitative real-time RT-PCR}

To verify the chip data, seven genes were selected for real-time RT-PCR analysis. The primer sequences were designed by Primer Express 2.0 software according to seven target genes a2m, spp1, cypla1, jun, icam 1, g6pc, and trim 24 (GenBank number: NM_012488, NM_012881,NM_012540, NM_021835, NM_012967, NM_013098 and NM_001044266) and synthesized by Shanghai Generay Biotech Co., Ltd. The gene-specific primers were following: forward primer 5'-CGAACATCCGTAAACCCAAAGTC-3' and reverse primer 5'-GCTGAGTCCACCACCACCAA- GTC-3 for $a 2 m$, forward primer 5'-TGATGACGACGACGATGACGATGG-3' and reverse primer 5'-ACGCTGGGCAACTGGGATGACCTT-3 for spp1, forward primer 5'-AGGACAGGAGGCT- GGACGAGA-3' and reverse primer 5'-ATGGTGAATGGGACAAAGGAT-3' for cyplal, forward primer 5'-TGCAAAGATGGAAACGACCTT-3' and reverse primer 5'- GCCGTAGGCGCCACTCT-3' for jun, forward primer 5'-TCAAACGGGAGATGAATGGT-3' and reverse primer 5'-CCTCTGGCG- GTAATAGGTGT-3' for icaml, and forward primer 5'-CTCAGGAACGCCTTCTATG-3' and reverse primer 5'-ACGGAGCTGTTGCTGTAAT-3' for g6cp, forward primer 5'-CAGTGGGAGGGTCTTA- CAATC-3' and reverse primer 5'-CTGGCCAGGGTCTACACTTG-3' for trim 24. Firstly their total mRNA of liver tissues from 
regenerating liver was isolated at the 10 recovery time points. Of them, $2 \mu \mathrm{g}$ RNA was reverse-transcribed using random primers and reverse transcription kit (Promega). First-strand cDNA samples were subjected to quantitative PCR amplification by using SYBR ${ }^{\circledR}$ Green I on the Rotor-Gene 3000A (Corbett Robotics, Brisbane, Australia). All of the PCR cycling conditions were modified at $95^{\circ} \mathrm{C}$ for $2 \mathrm{~min}$, followed by 40 cycles of $95^{\circ} \mathrm{C}$ for $15 \mathrm{~s}, 60^{\circ} \mathrm{C}$ for $15 \mathrm{~s}$, and $72^{\circ} \mathrm{C}$ for $30 \mathrm{~s}$. Every sample was analyzed in triplicate. Standard curves were generated from five repeated ten-fold serial dilutions of cDNA, and the copy numbers of target genes in every milliliter of the sample were calculated according to their corresponding standard curves (Wang et al., 2010).

\subsection{Identification of rat liver regeneration-related genes and rat acute hepatic failure-related genes in ERK1/2 signaling pathway}

To identify genes associated with ERK1/2 signaling pathway, the phrase "ERK1/2 signaling pathway" was input into NCBI (www.ncbi.nlm.nih.gov) and RGD (rgd.mcw.edu) to collect its genes associated with rat, mouse and human. Then, these genes were further obtained according to the biological pathway maps embodied at the databases including GENMAPP (www. genmapp.org), KEGG (www.genome.jp/kegg/pathway.htmL) and BIOCARTA (www. biocarta. com/genes/index. asp), etc and reconfirmed with related articles (Wang et al., 2007; Doniger et al., 2003; Ogata et al., 1999; Osband et al.,1990). In confirming the genes related to rat liver regeneration (LR) and the genes related to AHF, the images above were converted into signal values using Affymetrix GCOS 1.4 software. The data of each array were initially normalized by scaling all signals to a target intensity of 200. When $P$ value is $<0.05$, it means that the gene is present $(\mathrm{P})$, when $<0.065$, means marginal $(\mathrm{M})$, and when $>0.065$, means marked absent (A). Then, the signal value of each chip was normalized, and the relative values of genes were evaluated with the ratios comparing the normalized $\mathrm{P}$ value in $\mathrm{PH}$ groups to that in control groups. For example, the expression of genes with a relative value $\geq 3$ is considered up-regulated, the expression of genes with a relative value $\leq 0.33$ is considered as down-regulated, and genes with a relative value of $0.33 \sim 2.99$ are considered as unmeaning genes. To minimize the technical errors from microarray analysis, each sample was analyzed at least three times with Rat Genome 2302.0 microarray. Their average value was calculated as a corrective value. The genes which have expressed significantly during $\mathrm{CCl}_{4}$-induced AHF were considered as AHF-related genes, and the genes which have been significantly changed during LR with significant difference $(0.01 \leq P<0.05)$ or extremely significant difference $(P \leq 0.01)$ between $\mathrm{PH}$ and $\mathrm{OC}$, as LR-related genes.

\subsection{Synergy analysis of ERK1/2 signaling pathway-related genes}

A mathematical model $\left(\mathrm{E}_{\mathrm{t}}\right)$ was established to describe how physiological activities are governed by gene synergy (Xu \& Zhang, 2009), in accordance with the gene expression abundance of ERK1/2 signaling pathway in regenerating liver tissues detected by Rat Genome 2302.0 Array, the multivariate statistics (Chalmers et al.,1998), time series analysis (McCubrey et al., 2007), correlation analysis $\left(r_{\mathrm{ik}}\right)$ (Liu et al., 2009), and the principle that gene synergy governs physiological activity:

$$
\mathrm{E}_{\mathrm{t}}=\frac{\sum_{\mathrm{i}=1}^{\mathrm{n}} \sum_{\mathrm{k}=1}^{\mathrm{n}}\left[\left(\mathrm{X}_{\mathrm{i}}^{(\mathrm{t})}+\mathrm{X}_{\mathrm{k}}^{(\mathrm{t})}\right) * \mathrm{r}_{\mathrm{ik}}\right]}{\mathrm{n}(\mathrm{n}+1)}
$$

In the formula, the genes correlation coefficient $\left(\mathrm{r}_{\mathrm{ik}}\right)$ was defined as:

$$
\mathbf{r}_{i k}=\frac{m\left(\sum_{t=1}^{m} X_{i}^{(t)} X_{k}^{(t)}\right)-\left(\sum_{t=1}^{m} X_{i}^{(t)}\right)\left(\sum_{t=1}^{m} X_{k}^{(t)}\right)}{\sqrt{\left[m \sum_{t=1}^{m} X_{i}^{(t)}-\left(\sum_{t=1}^{m} X_{i}^{(t)}\right)^{2}\right]\left[m \sum_{t=1}^{m} X_{k}^{(t)^{2}}-\left(\sum_{t=1}^{m} X_{k}^{(t)}\right)^{2}\right]}}
$$

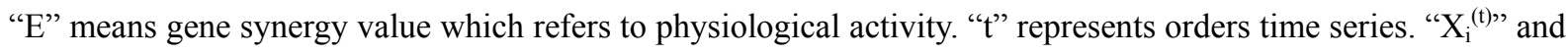
" $\left.\mathrm{X}_{\mathrm{k}}{ }_{\mathrm{k}} \mathrm{t}\right)$ " abundances of gene at $\mathrm{t}$. " $n$ " the total number of genes. Physiological activities in LR are more strengthened than in control when $\mathrm{E}_{\mathrm{t}^{-}} \mathrm{E}_{0}>\mathrm{E}_{0}$, more weakened when $\mathrm{E}_{\mathrm{t}}-\mathrm{E}_{0}<0$, similar in the two groups when $\mathrm{E}_{\mathrm{t}}-\mathrm{E}_{0}=0$. 


\section{Results}

\subsection{The expression profiles of ERK1/2 signaling pathway-related genes in rat liver regeneration and in rat acute} hepatic failure

The data from NCBI, RGD, etc and biological pathway maps in GENMAPP, KEGG and BIOCARTA etc showed that 165 genes were related to ERK1/2 signaling pathway. Of them, 161 genes were in Rat Genome 2302.0 Array. This array was used to detect expression changes of genes related to ERK1/2 signaling pathway in rat liver regeneration (LR) and in rat acute hepatic failure (AHF), showing that 46 genes were significantly expressed. In detail, 36 genes were the LR-specific genes, including 29 up-regulated and 7 down-regulated genes. 24 genes were the AHF-specific genes, including 18 up-regulated and 6 down-regulated genes. And 14 genes were the common genes. Of them, 9 genes were up-regulated and 2 genes were down-regulated in both LR and AHF, 2 genes were up-regulated in LR and down-regulated in AHF, one gene was down-regulated in LR and up-regulated in AHF (Table 1, Figure 1). To confirm the reliability of the array detection results, real-time RT-PCR was used to detect the expression changes of genes including a2m, spp1, cyplaland trim 24 in LR and AHF, and it was founded that the gene expression trends of these genes detected by RT-PCR and Rat Genome 2302.0 Array were generally consistent, suggesting that the array check results were reliable.

\subsection{Comparative analysis of the detection results of real-time polymerase chain reaction and that of microarray}

To confirm the results of the microarray analysis, RT-PCR assays were used to detect the expression changes of the genes including a2m, sppl, cyplal and trim 24 at 3, 6, 12, 24, 48 and $72 \mathrm{~h}$ after $\mathrm{CCl}_{4}$ treatment and jun, icaml, g6pc, and trim 24 in regenerating livers at $0,2,6,12,24,30,36,72,120$ and $168 \mathrm{~h}$ after PH. The result showed that relative abundance of mRNA were not all the same, but expression trends of the genes detected by above two methods were generally consistent, suggesting that the array detection results were reliable (Figure 2).

3.3 The physiological activities uncovered by expression profiles of ERK1/2 signaling pathway-related genes in rat liver regeneration and rat acute hepatic failure

The gene synergy and the roles of the fourteen paths of ERK1/2 signaling pathway in rat LR and rat AHF were analyzed by using the mathematical model above, and the results were listed blow. Gene synergy value $\left(E_{t}\right)$ of cell proliferation-promoting path 4 was smaller than that of control at $6 \mathrm{~h}$ of rat AHF occurrence, and that of path 13 was smaller at $24 \mathrm{~h}$ of rat $\mathrm{LR}$. But $\mathrm{E}_{\mathrm{t}}$ of cell proliferation-promoting paths 2, 8, 9 and 11 were greater at $12 \mathrm{~h}$ of rat $L R$, and $E_{t}$ of cell proliferation-promoting path 11 were greater at $12 \mathrm{~h}$ of rat AHF occurrence. $E_{t}$ of all paths were similar to that of the control at $24 \mathrm{~h}$ of rat AHF occurrence and $6 \mathrm{~h}, 72 \mathrm{~h}$ of rat LR. $\mathrm{E}_{t}$ of cell proliferation-promoting paths 2 and 6 were greater at 12h of rat AHF occurrence. However, paths 1, 3-5, 7, 10, 12 and 14 were not found to participate in LR and AHF occurrence (Figure 3).

\section{Discussion}

It was reported that 2-6h after rat partial hepatectomy $(\mathrm{PH})$ is the priming phase of rat liver regeneration (LR). Hepatocytes were activated at $2 \mathrm{~h}$ after $\mathrm{PH}$, and their transition from G0 phase to cell cycle occurred at 2-6h after PH (Guo et al., 2006; Xu et al., 2010). On the other hand, liver tissue was infiltrated with inflammatory cells, and necrosis occurred to some hepatocytes at $6 \mathrm{~h}$ of rat acute hepatic failure (AHF) (Leu et al., 2003). Gene synergy value $\left(\mathrm{E}_{\mathrm{t}}\right)$ of all paths of ERK1/2 signaling pathway were similar to those of the control at $6 \mathrm{~h}$ of LR, indicating that cell proliferation-promoting activities occurred later in LR. $E_{t}$ of path 4 which promoted cell proliferation at 6h of AHF was smaller than that of control, indicating that this path may inhibit cell proliferation, which is consistent with the actual physiological activities at this time point. It is inferred that ERK1/2 signaling pathway is involved in regulating cell proliferation of AHF at this time point, but not in that of LR.

It was shown that 6-12h after rat PH is G1 phase of hepatocytes (Xu et al., 2010). Inflammatory response of liver was strengthened, liver lobule got damaged, and hepatocytes took on necrosis and apoptosis at $12 \mathrm{~h}$ of AHF (Mookerjee et al., 2007). $\mathrm{E}_{\mathrm{t}}$ of path 2, 8 and 9 which promoted cell proliferation and path 11 which inhibited cell proliferation were greater than that of control at $12 \mathrm{~h}$ of LR, implying that path 2, 8 and 9 promote cell proliferation and path 11 inhibits cell proliferation at this time point. $\mathrm{E}_{\mathrm{t}}$ of path 11 which inhibited cell proliferation was greater at $12 \mathrm{~h}$ of AHF, suggesting that this path inhibit cell proliferation at this time point, which agrees with the actual physiological activities. It was concluded that ERK1/2 signaling pathway is involved in the regulation of cell proliferation of LR and AHF at this time point.

It was found that $12-24 \mathrm{~h}$ after rat PH is S phase of hepatocytes (Xu et al., 2010). Immune and inflammatory responses of liver were strengthened, apoptosis of liver cells continued, and the liver injury got severely at $24 \mathrm{~h}$ of AHF occurrence (Mookerjee et al., 2007). $E_{t}$ of path 13 which inhibited cell proliferation was smaller than that of control at $24 \mathrm{~h}$ of rat LR in this study, meaning that the cell proliferation-inhibiting effect of this path is 
more weakened at this time point. $E_{t}$ of all the paths were similar to those of the control at $24 \mathrm{~h}$ of AHF, suggesting that cell proliferation does not start at this time point, which agrees with the actual physiological activities. The results above revealed that ERK1/2 signaling pathway is involved in cell proliferation of LR at this time point, but not in that of AHF.

Generally, 36-72h after rat PH is the second cell cycle of hepatocytes (Xu et al., 2010). Inflammatory response and cell apoptosis of liver were decreased, cell proliferation and differentiation of liver were increased, and liver function began to recover at 72h of AHF occurrence (Zhang et al., 2005; Pathikonda et al., 2010). $\mathrm{E}_{\mathrm{t}}$ of all the paths were similar to those of the control at $72 \mathrm{~h}$ of LR, implying that the paths above is not involved in cell proliferation of second cell cycle of hepatocytes at this time point. $E_{t}$ of path 2 and 6 which promoted cell proliferation were greater, indicating that these two paths promote cell proliferation at this time point, which is in accordance with the actual physiological activities. It is obvious that ERK1/2 signaling pathway is involved in regulating cell proliferation of AHF at this time point, but not in that of LR.

In summary, Rat Genome 2302.0 Array was used to detect the gene expression profiles of ERK1/2 signaling pathway in rat LR and rat AHF occurrence, and bioinformatics and systems biology methods to analyze the roles of fourteen paths of ERK1/2 signaling pathway in regulating rat LR and rat AHF occurrence in this study. It was found that seven paths of ERK1/2 signaling pathway participated in regulating cell proliferation and apoptosis of rat LR and rat AHF occurrence. The results above will be confirmed by using gene addition and gene interference in vivo and in vitro in the future.

\section{References}

Chalmers, J. J., Zborowski, M., Sun, L. \& Moore, L. (1998). Flow through, immunomagnetic cell separation. Biotechnol Prog, 14, 141-8. http://dx.doi.org/10.1021/bp9701401

Cheng, W. T., \& Tian, D. Y. (2010). Effects and mechanism of endotoxemia on carbohydrate metabolism in D-GalN-reduced acute hepatic failure rats. Chinese Journal of Integrated Traditional and Western Medicine, 20, 38-40. http://dx.doi.org/10.3969/j.issn.1005-0264.2010.01.013

Doniger, S. W., Salomonis, N., Dahlquist, K. D., Vranizan, K., Lawlor, S. C. \& Conklin, B. R.(2003). MAPPFinder: using Gene Ontology and GenMAPP to create a global gene-expression profile from microarray data. Genome Biol, 4, R7. http://dx.doi.org/10.1186/gb-2003-4-1-r7

Fausto, N. (2000). Liver regeneration. J Hepato, 32, 19-31. http://dx.doi.org/10.1016/S0168-8278(00)80412-2

Felipo, V. \& Butterworth, R. F. (2002). Mitochondrial dysfunction in acute hyperammonemia. Neurochem Int, 40, 487-491. http://dx.doi.org/10.1016/S0197-0186(01)00119-X

Guo, F., Nian, H., Zhang, H., Huang, L., Tang, Y., Xiao, X. \& He, D. (2006). Proteomic analysis of the transition from quiescent to proliferating stages in rat liver hepatectomy model. Proteomics, 6, 3075-3086. http://dx.doi.org/10.1002/pmic.200500322

Higgins, G. M. \& Anderson, R. M. (1931). Experimental pathology of the liver: restoration of the liver of the white rat following partial surgical removal. Arch Pathol, 1, 186-202.

Huh, C. G., Factor, V. M., Sánchez, A., Uchida, K., Conner, E. A. \& Thorgeirsson, S. S. (2004). Hepatocyte growth factor/c-met signaling pathway is required for efficient liver regeneration and repair. Proc Natl Acad Sci USA, 101, 4477-4482. http://dx.doi.org/10.1073/pnas.0306068101

Kant, S., Schumacher, S., Singh, M. K., Kispert, A., Kotlyarov, A. \& Gaestel, M. (2006). Characterization of the atypical MAPK ERK4 and its activation of the MAPK-activated protein kinase MK5. Biol Chem, 281, 35511-35519. http://dx.doi.org/10.1074/jbc.M606693200

Leu, J. I., Crissey, M. A. \& Taub, R. (2003). Massive hepatic apoptosis associated with TGF-beta1 activation after Fas ligand treatment of IGF binding protein-1-deficient mice. $J$ Clin Invest, 111, 129-139. http://dx.doi.org/10.1172/JCI200316712

Leu, J. I., Crissey, M. A., Craig, L. E. \& Taub, R. (2003). Impaired hepatocyte DNA synthetic response posthepatectomy in insulin-like growth factor binding protein 1-deficient mice with defects in C/EBP $\beta$ and mitogen-activated protein kinase/extracellular signal-regulated kinase regulation. Mol Cell Biol, 23, 1251-1259. http://dx.doi.org/10.1128/MCB.23.4.1251-1259.2003

Liu, S. P., Wang, L., Xue, Y. H. \& Shou, H. X. (2009). Research progress in ARF-GEF gene family. Yi Chuan, 31, 982-92. http://dx.doi.org/10.3724/SP.J.1005.2009.00982

McCubrey, J. A., Steelman, L. S., Abrams, S. L., et al. (2007). Roles of the Raf/MEK/ERK pathway in cell 
growth, malignant transformation and drug resistance. Biochim Biophys Acta, 1773, 1263-84. http://dx.doi.org/10.1016/j.advenzreg.2006.01.004

Michalopoulos, G. K. (2010). Liver regeneration after partial hepatectomy: critical analysis of mechanistic dilemmas. Am J Pathol, 176, 2-13 http://dx.doi.org/10.2353/ajpath.2010.090675

Mookerjee, R. P., Dalton, R. N., Davies, N. A., Hodges, S. J., Turner, C., Williams, R. \& Jalan, R. (2007). Inflammation is an important determinant of levels of the endogenous nitric oxide synthase inhibitor asymmetric dimethylarginine (ADMA) in acute liver failure. Liver Transpl, 13, 400-405. http://dx.doi.org/10.1002/lt.21053

Murata, S., Ohkohchi, N., Matsuo, R., Ikeda, O., Myronovych, A. \& Hoshi, R. (2007). Platelets promote liver regeneration in early period after hepatectomy in mice. World $J$ Surg, 31, 808-816. http://dx.doi.org/10.1007/s00268-006-0772-3

Ogata, H., Goto, S., Sato, K., Fujibuchi, W., Bono, H. \& Kanehisa, M. (1999). KEGG: Kyoto Encyclopedia of Genes and Genomes. Nucleic Acids Res, 27, 29-34.

Osband, M. E. \& Cashon, G. W. (1990). Biocare: biotechnology in the clinical practice of medicine. Clin Res, 38, 5-9.

Pathikonda, M., \& Munoz, S. J. (2010). Acute liver failure. Ann Hepatol, 9, 7-14.

Rescan, C., Coutant, A., Talarmin, H., et al. (2010). Mechanism in the sequential control of cell morphology and $\mathrm{S}$ phase entry by epidermal growth factor involves distinct MEK/ERK activations. Mol Biol Cell, 12, 725-738

Rose, B. A., Force, T. \& Wang, Y. (2010). Mitogen-activated protein kinase signaling in the heart: angels versus demons in a heart-breaking tale. Physiol Rev, 90, 1507-1546. http://dx.doi.org/10.1152/physrev.00054.2009

Talarmin, H., Rescan, C., Cariou, S., et al. (1999). The Mitogen-Activated Protein Kinase Kinase/Extracellular Signal-Regulated Kinase Cascade Activation Is a Key Signalling Pathway Involved in the Regulation of G1 Phase Progression in Proliferating Hepatocytes. Molecular And Cellular Biology, 19, 6003-6011.

Wang, G. P. \& Xu, C. S. (2010). Reference gene selection for real-time rt-PCR in eight kinds of rat regenerating hepatic cells. Mol Biotechnol, 46, 49-57. http://dx.doi.org/10.1007/s12033-010-9274-5

Wang, J. Z., Du, Z., Payattakool, R., Yu, P. S. \& Chen, C. F. (2007). A new method to measure the semantic similarity of GO terms. Bioinformatics, 23, 1274-1281. http://dx.doi.org/10.1093/bioinformatics/btm087

Xu, C. S. \& Chang, C. F. (2008). Expression profiles of the genes associated with metabolism and transport of amino acids and their derivatives in rat liver regeneration. Amino Acids, 34, 91-102. http://dx.doi.org/10.1007/s00726-007-0576-2

Xu, C. S. \& Zhang, J. B. (2009). Research on the Functional Genomics of the Rat Regenerating Liver. Beijing: Higher Education Press.

Xu, C. S., Chen, X. G., Chang, C. F., et al. (2010). Transcriptome analysis of hepatocytes after partial hepatectomy in rats. Dev Genes Evol, 220, 263-274. http://dx.doi.org/10.1007/s00427-010-0345-1

Yokoyama, Y., Nagino, M. \& Nimura, Y. (2007). Mechanisms of hepatic regeneration following portal vein embolization and partial hepatectomy: a review. World $J$ Surg, 31, 367-374. http://dx.doi.org/10.1007/s00268-006-0526-2

Zhang, L. M., Liu, D. W., Liu, J. B., Zhang, X. L., Wang, X. B., Tang, L. M. \& Wang, L. Q. (2005). Effect of naked eukaryotic expression plasmid encoding rat augmenter of liver regeneration on acute hepatic injury and hepatic failure in rats. World J Gastroenterol, 11, 3680-3685. 
Table 1. Expression changes of ERK1/2 signaling pathway-related genes in rat liver regeneration and in rat acute hepatic failure

\begin{tabular}{|c|c|c|c|c|c|c|c|c|c|c|c|c|c|c|c|c|c|c|c|c|c|c|}
\hline \multirow{2}{*}{ Protien/Gene } & \multicolumn{5}{|c|}{ Recovery time (h) after PH } & \multicolumn{6}{|c|}{ Recovery time (h) after AHF } & \multirow{2}{*}{ Protien/Gene } & \multicolumn{5}{|c|}{ Recovery time (h) after $\mathrm{PH}$} & \multicolumn{5}{|c|}{ Recovery time (h) after AHF } \\
\hline & 2 & 6 & 12 & 24 & 72 & 3 & 6 & 12 & 24 & & 72 & & 2 & 6 & 12 & 24 & 72 & 3 & 6 & 12 & 24 & 72 \\
\hline \multicolumn{12}{|c|}{ Pathway 1 : G-protein $\rightarrow \mathrm{PKC}_{\rightarrow}$ Ras } & \multicolumn{6}{|c|}{ Pathway 5 : integrins $\rightarrow$ Grb2 $\rightarrow$ Ras } & & & & & \\
\hline (1) GQ/GNA14 & & & $\uparrow$ & $\uparrow$ & $\uparrow$ & & & & & & & (6) MAP2KLIP1/MAPK3 & & & & & $\uparrow$ & & & & & \\
\hline IGNA15 & & & $\uparrow$ & & $\uparrow$ & & & & & & & Pathway 6 : integrins $\rightarrow$ FAK & $\rightarrow \operatorname{Rac}$ & & & & & & & & & \\
\hline /GNG2 & & & & & $\uparrow$ & & & & & & & (9) INTEGRINS/ITGB2 & & & & & $\uparrow$ & & & & & \\
\hline /GNGT2 & & & & & $\uparrow$ & & & & & & & (4) PYK2/PTK2B & & $\uparrow$ & & & $\uparrow$ & & & & & $\uparrow$ \\
\hline (2) PLC /PLCB2 & $\downarrow$ & & & & & & & & & & & (10) PAK/PAK3 & & & & $\downarrow$ & $\downarrow$ & & & & & \\
\hline (3) $\mathrm{PKC} / \mathrm{PRKCQ}$ & & $\uparrow$ & $\uparrow$ & & & & & & & & & (6) MAP2KLIP1/MAPK3 & & & & & $\uparrow$ & & & & & \\
\hline IPRKCZ & & $\uparrow$ & $\uparrow$ & $\uparrow$ & $\uparrow$ & & & & & & & Pathway 7 : G-protein $\rightarrow$ PI3K & $\mathrm{K}_{\rightarrow} \mathrm{Ra}$ & & & & & & & & & \\
\hline (4) PYK2/PTK2B & & $\uparrow$ & & & $\uparrow$ & & & & & & $\uparrow$ & (1) $G Q / G N G T 2$ & & & & & $\uparrow$ & & & & & \\
\hline (5) SRC/SRC & & $\uparrow$ & $\uparrow$ & & $\uparrow$ & & & & & & & (11) PI3K/PIK3C2G & & & & & $\downarrow$ & & & $\downarrow$ & $\downarrow$ & \\
\hline \multicolumn{3}{|c|}{ (6) MAP2KLIP1/MAPK3 } & & & $\uparrow$ & & & & & & & (10) PAK/PAK3 & & & & $\downarrow$ & $\downarrow$ & & & & & \\
\hline \multirow{2}{*}{\multicolumn{3}{|c|}{$\begin{array}{l}\text { Pathway } 2: \mathrm{Ca}_{2}+{ }_{\rightarrow} \mathrm{PKC}_{\rightarrow} \text { Ras } \\
\text { (7) } \mathrm{CACN} / \mathrm{CACNA} 1 \mathrm{~F}\end{array}$}} & & $\uparrow$ & & & & & & & & \multicolumn{3}{|c|}{ Pathway $8:$ : G-protein $\rightarrow$ EPAC $_{\rightarrow}$ Rap1 } & & & $\uparrow$ & & & & & \\
\hline & & & & 1 & & & & $\uparrow$ & & & & (1) GQ/GNAL & $\rightarrow T$ & dapl & $\uparrow$ & & & & & & & \\
\hline /CACNA1H & $\uparrow$ & $\uparrow$ & & $\uparrow$ & & $\uparrow$ & $\uparrow$ & & & & & (12) $\mathrm{AC} / \mathrm{ADCY} 3$ & & & $\uparrow$ & & $\uparrow$ & $\downarrow$ & & & $\downarrow$ & \\
\hline /CACNA1I & & & $\uparrow$ & & & & & & & & & IADCY5 & & & & & & & $\uparrow$ & $\uparrow$ & & \\
\hline /CACNB1 & $\uparrow$ & & & & & & $\uparrow$ & & & & & /ADCY8 & $\uparrow$ & $\uparrow$ & $\uparrow$ & $\uparrow$ & $\uparrow$ & $\uparrow$ & $\uparrow$ & $\uparrow$ & $\uparrow$ & \\
\hline /CACNB4 & & & & & $\downarrow$ & & $\uparrow$ & $\uparrow$ & $\uparrow$ & & $\uparrow$ & /ADCY9 & $\downarrow$ & & & & & & & & & \\
\hline /CACNG1 & & & & & & $\uparrow$ & & $\uparrow$ & & & & (6) MAP2KLIP1/MAPK3 & & & & & $\uparrow$ & & & & & \\
\hline /CACNG2 & $\uparrow$ & $\uparrow$ & $\uparrow$ & $\uparrow$ & $\uparrow$ & & & & & & & Pathway 9 : G-protein $\rightarrow$ PKA & $\mathrm{A}_{\rightarrow} \mathrm{Ra}$ & & & & & & & & & \\
\hline /CACNG3 & & & $\uparrow$ & & & $\uparrow$ & & & & & & (1) $G Q / G N A L$ & & & $\uparrow$ & & & & & & & \\
\hline /CACNG6 & $\uparrow$ & $\uparrow$ & $\uparrow$ & $\uparrow$ & $\uparrow$ & & $\uparrow$ & & & & & (12) $A C / A D C Y 3$ & & & $\uparrow$ & & $\uparrow$ & $\downarrow$ & & & $\downarrow$ & \\
\hline /CACNG8 & $\uparrow$ & & $\uparrow$ & $\uparrow$ & $\uparrow$ & & & & & & & /ADCY5 & & & & & & & $\uparrow$ & $\uparrow$ & & \\
\hline (3) $P K C / P R K C Q$ & & $\uparrow$ & $\uparrow$ & & & & & & & & & /ADCY8 & $\uparrow$ & $\uparrow$ & $\uparrow$ & $\uparrow$ & $\uparrow$ & $\uparrow$ & $\uparrow$ & $\uparrow$ & $\uparrow$ & \\
\hline IPRKCZ & & $\uparrow$ & $\uparrow$ & $\uparrow$ & $\uparrow$ & & & & & & & /ADCY9 & $\downarrow$ & & & & & & & & & \\
\hline (5) $\mathrm{SRC} / \mathrm{SRC}$ & & $\uparrow$ & $\uparrow$ & & $\uparrow$ & & & & & & & (6) MAP2KLIP1/MAPK3 & & & & & $\uparrow$ & & & & & \\
\hline (4) PYK2/PTK2B & & $\uparrow$ & & & $\uparrow$ & & & & & & $\uparrow$ & Pathway $10:$ Mos $_{\rightarrow}$ MEK1/2 & & & & & & & & & & \\
\hline (6) MAP2KLIP1/MAPK & & & & & $\uparrow$ & & & & & & & (13) MOS/MOS & & & & & & $\uparrow$ & & & & \\
\hline Pathway $3: R^{2} K_{\rightarrow}$ PKC & $\rightarrow \mathrm{Ra}$ & & & & & & & & & & & Pathway 11 : G-protein $\rightarrow$ PK & $\langle A-C$ & -Raf & & & & & & & & \\
\hline (8) RTK/KDR & & & & & & & & & $\downarrow$ & & & (1) $G Q / G N A L$ & & & $\uparrow$ & & & & & & & \\
\hline NTRK 1 & & & & & & $\uparrow$ & & & & & & (12) $A C / A D C Y 3$ & & & $\uparrow$ & & $\uparrow$ & $\downarrow$ & & & $\downarrow$ & \\
\hline /PDGFRB & & & $\uparrow$ & & & & & & & & & IADCY5 & & & & & & & $\uparrow$ & $\uparrow$ & & \\
\hline /FGFR2 & & & & $\uparrow$ & $\uparrow$ & & & & & & & /ADCY8 & $\uparrow$ & $\uparrow$ & $\uparrow$ & $\uparrow$ & $\uparrow$ & $\uparrow$ & $\uparrow$ & $\uparrow$ & $\uparrow$ & \\
\hline MET & & & & $\uparrow$ & & & $\downarrow$ & $\downarrow$ & $\downarrow$ & & & /ADCY9 & $\downarrow$ & & & & & & & & & \\
\hline (4) PYK2/PTK2B & & $\uparrow$ & & & $\uparrow$ & & & & & & $\uparrow$ & (6) MAP2KLIP1/MAPK3 & & & & & $\uparrow$ & & & & & \\
\hline (3) PKC/PRKCQ & & $\uparrow$ & $\uparrow$ & & & & & & & & & Pathway 12 : NF1-Ras & & & & & & & & & & \\
\hline IPRKCZ & & $\uparrow$ & $\uparrow$ & $\uparrow$ & $\uparrow$ & & & & & & & (14) NF1/NF1 & $\uparrow$ & $\uparrow$ & $\uparrow$ & & & $\uparrow$ & & & & \\
\hline (5) SRC/SRC & & $\uparrow$ & $\uparrow$ & & $\uparrow$ & & & & & & & Pathway 13 : PP1/2 - MEK & & & & & & & & & & \\
\hline (6) MAP2KLIP1/MAPK & & & & & $\uparrow$ & & & & & & & (15) PP1/2/PPP1R1B & $\uparrow$ & $\uparrow$ & $\uparrow$ & & & & & & & \\
\hline Pathway $4:$ RTK $\rightarrow$ Grb2 & $\rightarrow \mathrm{Ra}$ & & & & & & & & & & & IPPP1R3A & & $\uparrow$ & & $\uparrow$ & & $\uparrow$ & & & & \\
\hline (8) RTK/KDR & & & & & & & & & $\downarrow$ & & & /PPP1R3B & $\downarrow$ & $\downarrow$ & $\downarrow$ & & $\downarrow$ & & $\downarrow$ & $\downarrow$ & $\downarrow$ & \\
\hline /NTRK1 & & & & & & $\uparrow$ & & & & & & /PPP1R3C & $\downarrow$ & $\downarrow$ & & & & & & & & \\
\hline /PDGFRB & & & $\uparrow$ & & & & & & & & & /PPP1R10 & & $\uparrow$ & & & & & & & & \\
\hline /FGFR2 & & & & $\uparrow$ & $\uparrow$ & & & & & & & /PPP1R14B & & & & & & & & & $\uparrow$ & \\
\hline MET & & & & $\uparrow$ & & & $\downarrow$ & $\downarrow$ & $\downarrow$ & & & /PPP2R2A & $\uparrow$ & $\uparrow$ & $\uparrow$ & & & $\uparrow$ & $\uparrow$ & $\uparrow$ & & \\
\hline (6) MAP2KLIP1/MAPK & & & & & $\uparrow$ & & & & & & & IPPP2R2B & & & & & & $\downarrow$ & & & & \\
\hline Pathway 5 : integrins $\rightarrow$ & Grb2 & Ras & & & & & & & & & & /PPP2CA & & & & & & & $\uparrow$ & $\uparrow$ & & \\
\hline (9) INTEGRINS/ITGB2 & & & & & $\uparrow$ & & & & & & & (6) MAP2KLIP1/MAPK3 & & & & & $\uparrow$ & & & & & \\
\hline (5) SRC/SRC & & $\uparrow$ & $\uparrow$ & & $\uparrow$ & & & & & & & Pathway 14 : MKP - ERK1 & & & & & & & & & & \\
\hline (4) PYK2/PTK2B & & $\uparrow$ & & & $\uparrow$ & & & & & & $\uparrow$ & (16) MKPS/DUSP1 & & & & & & $\uparrow$ & & & & \\
\hline
\end{tabular}

$* \uparrow$ up-regulation, $\downarrow$ down-regulation. 
Figure 1

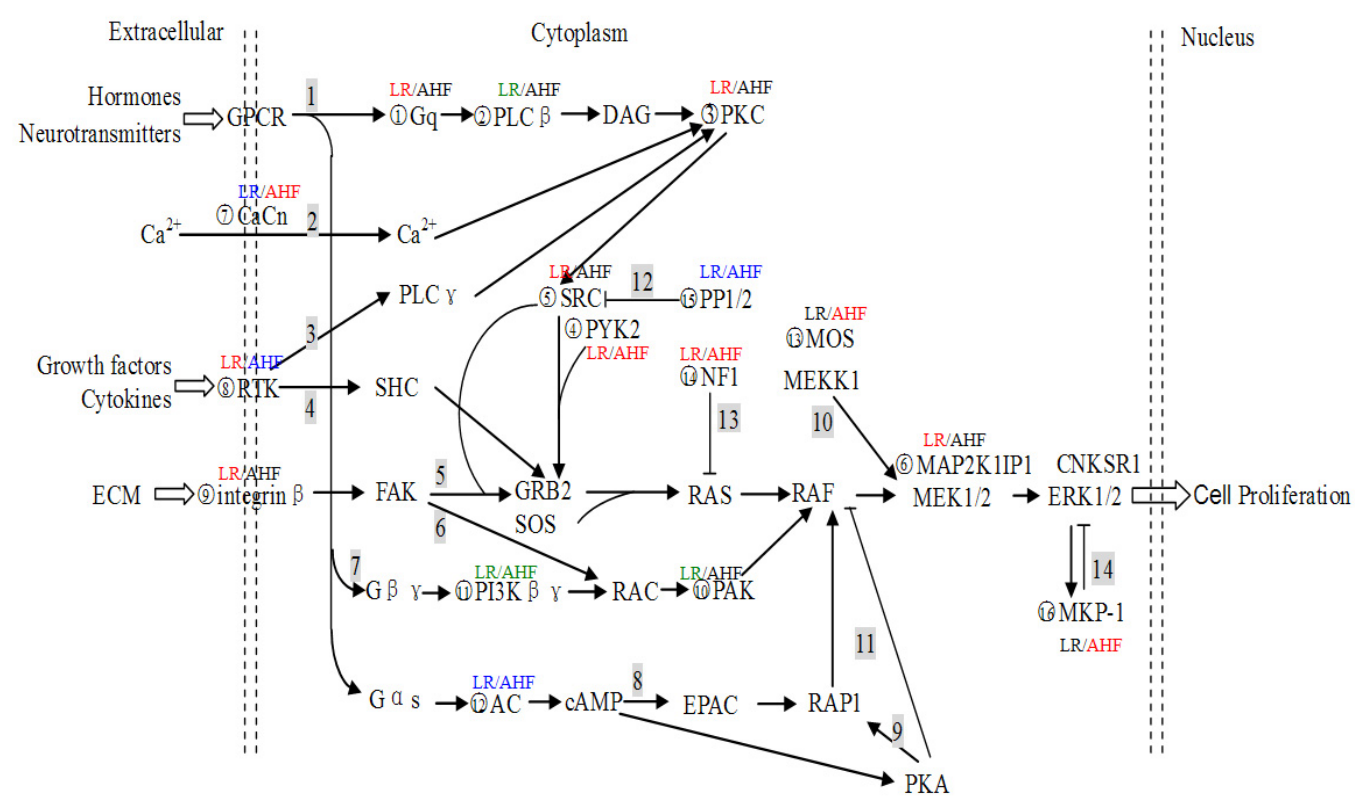

Figure 1. Fourteen path of ERK1/2 signaling pathway and genes related to these paths

Symbols in red mean the up-regulated genes, those in green the down-regulated, and those with yellow ground the up/down-regulated genes. The figures under grey ground present the ordinal numbers of the paths, and the figures in circle the ordinal numbers of proteins which share the same ordinal numbers in the table above. 


\section{Figure 2}
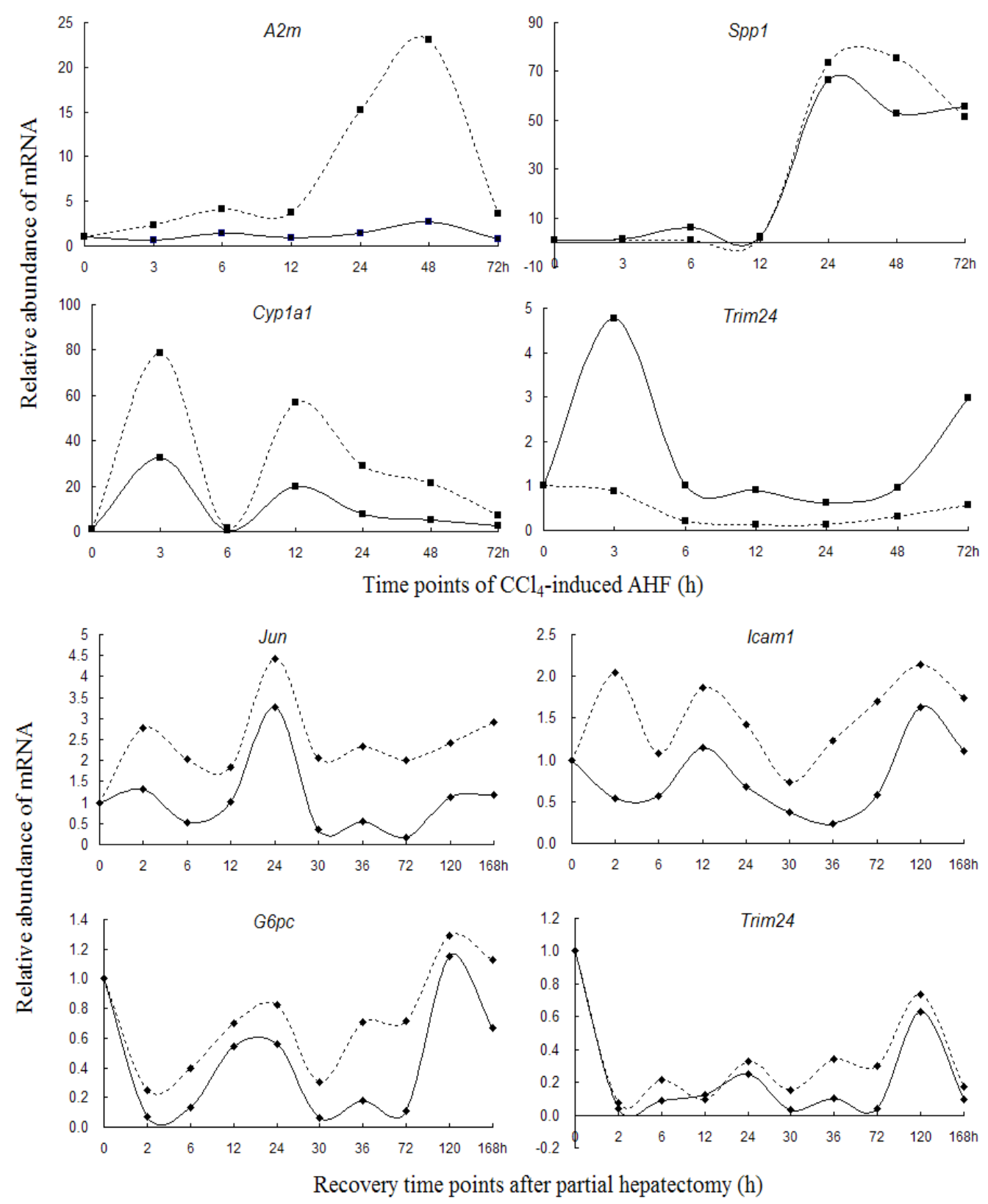

Figure 2. mRNA expression of four selected genes measured by microarrays and RT-PCR. Solid line presented the results of RT-PCR and dotted line that of Rat Genome 230 2.0 Array 


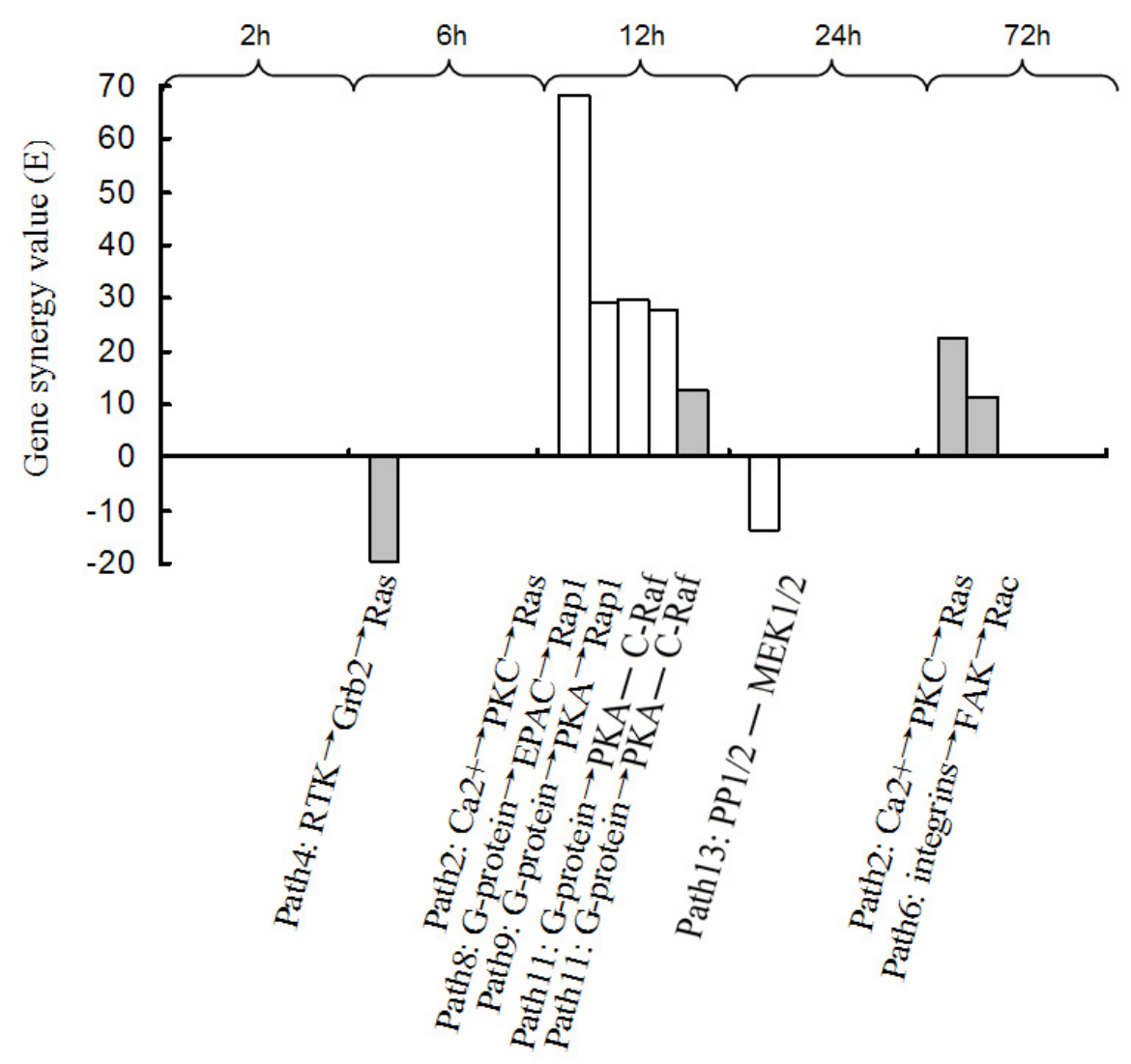

Figure 3. Cell proliferation regulated by thirty eight paths of ERK1/2 signaling pathway in rat liver regeneration (LR) and rat acute hepatic failure (AHF). X-axis: Path of ERK1/2 signaling pathway and time points of LR/AHF. LR-related path; AHF-related path 